\title{
Spectral variation of BU Tau
}

\author{
Kalju Annuk \\ Tartu Observatory, \\ 61602 Tõravere, Estonia \\ email: annuk@aai.ee
}

\begin{abstract}
We present the preliminary result of our spectroscopic observations covering a period over more than ten years, from 1999 up to 2010. We analyze line profile variations of several lines before the shell phase and during the shell phase. The measured radial velocity, equivalent width and line intensity variations are presented.
\end{abstract}

Keywords. stars: emission-line, Be, stars: individual (BU Tau), line: profiles

\section{Introduction}

BU Tau (Pleione) is well-known B/Be star located in the Pleiades cluster. It is accepted that this star has periodic ( $\mathrm{P} \sim 34$ years) shell formation. So far, there has been clearly observed two shell phases, in 1938-1954 and 1972-1988. The duration of shell phase lasts about 16 years. The third shell phase started in 2005-2006 and it has to be continued up to 2022. BU Tau is also known as a photometric variable. Although this object has been under observation and investigation more than 100 years, there are still several unsolved questions.

\section{Observations and reduction}

Observations were carried out with the $1.5 \mathrm{~m}$ telescope of the Tartu Observatory, using a Cassegrain spectrograph ASP-32. All observations were obtained with the $1800 \mathrm{l} / \mathrm{mm}$ grating. The nitrogen cooling CCD camera Orbis-1 (512x512 pixels) was used in 1999 up to March 2006. Starting from April 2006, the Peltier cooling CCD Andor Newton DU-970N (1600x200 pixels) has been used. The spectral resolution was $\sim 0.26 \AA /$ pixel (with Orbis-1) or $\sim 0.17 \AA /$ pixel (with Andor Newton) at the $\mathrm{H}_{\alpha}$. Three spectral regions were observed: around the $\mathrm{H}_{\alpha}, \mathrm{H}_{\beta}$ and $\lambda<4000 \AA$. The MIDAS package has been used for the reduction. All spectra were calibrated with a ThAr lamp and normalized to the continuum level. Radial velocities of the $\mathrm{H}_{\alpha}$ emission lines were measured by the bisector method and radial velocities of the other lines by fitting a gaussian profiles.

\section{Discussion}

The variation of the equivalent width $(\mathrm{EW})$ of the $\mathrm{H}_{\alpha}$ line is presented in the left part of Figure 1. We can see that the EW has continuously decreased from the value $\sim 35 \AA$ (November 1999) up to $\sim 25 \AA$ (March 2006). At that time the star was in a Be phase. During the next $\sim 450$ days the EW very quickly decreased reaching to its minimum value $\sim 5.5 \AA$. This period corresponds to the shell formation phase.

The central intensities of the $\mathrm{H}_{\alpha}$ and $\mathrm{H}_{\beta}$ absorption components (right part of Figure 1) behave in the same way as the equivalent width of the $\mathrm{H}_{\alpha}$ line. We have to mention that central intensities of the $\mathrm{H}_{\alpha}$ and $\mathrm{H}_{\beta}$ have a very good correlation. Starting the March 2006, the central intensity of the $\mathrm{H}_{\beta}$ line is smaller than 1.0. 

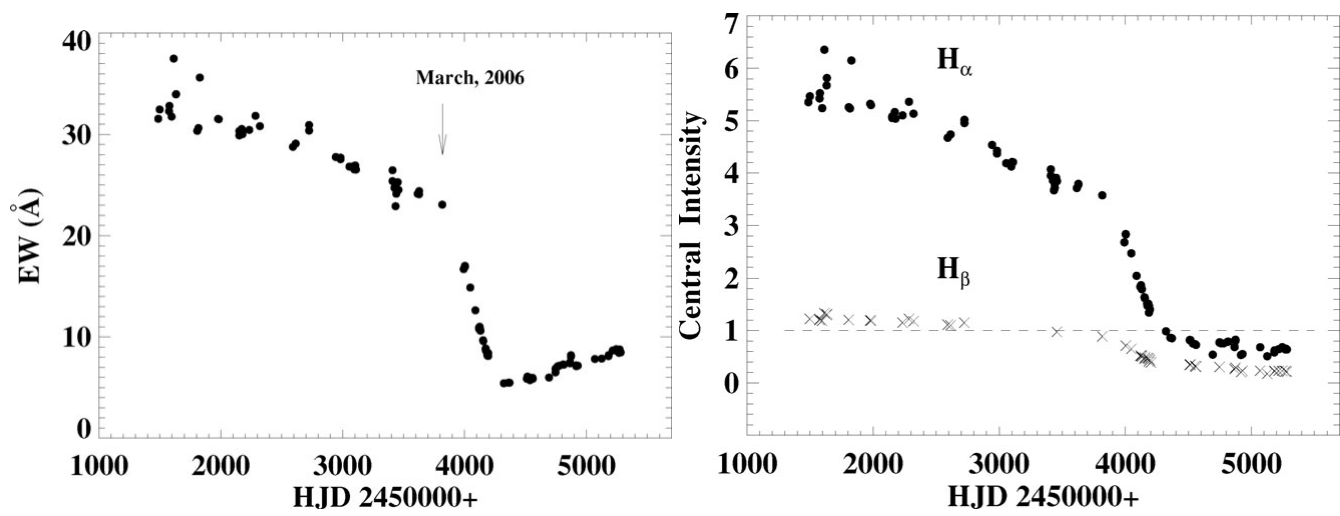

Figure 1. Left: The equivalent width variation of the $\mathrm{H}_{\alpha}$ line. Right: The central intensities of the $\mathrm{H}_{\alpha}$ and $\mathrm{H}_{\beta}$ absorption component.
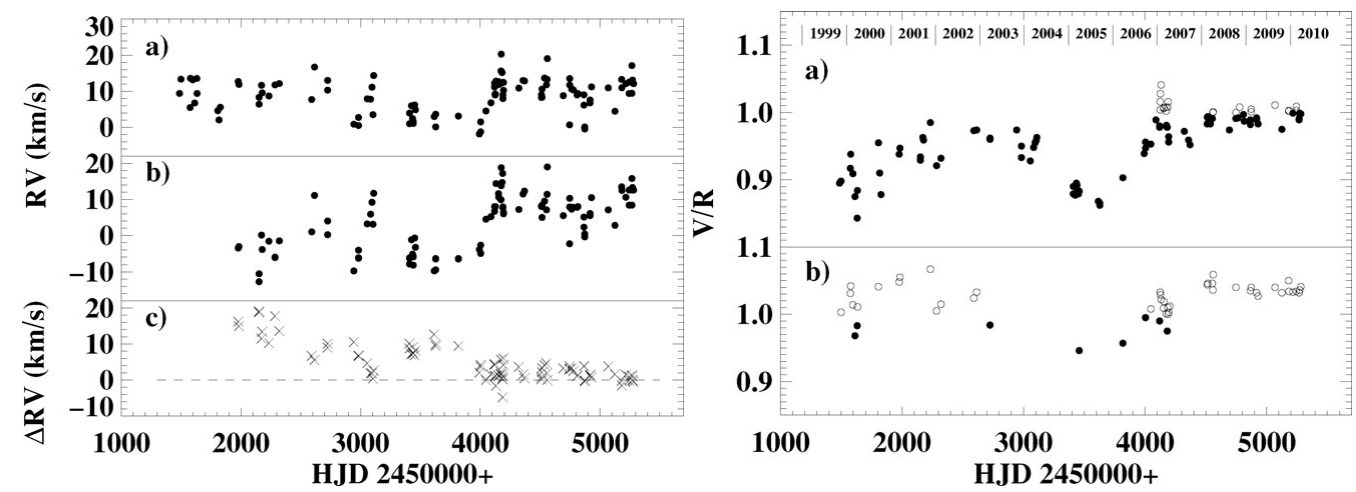

Figure 2. Left: The radial velocities of the $\mathrm{H}_{\alpha}$ : a) emission component $\left(\mathrm{RV}_{e m}\right)$; b) absorption component $\left(\mathrm{RV}_{a b s}\right)$; c) emission minus absorption. Right: The $\mathrm{V} / \mathrm{R}$ variations: a) of the $\mathrm{H}_{\alpha}$ line; b) of the $\mathrm{H}_{\beta}$ line.

The radial velocity variation of the $\mathrm{H}_{\alpha}$ is shown in the left part of Figure 2 . As the line profile is somewhat asymmetric then we used for the calculation of radial velocities of emission component the levels which correspond to the more symmetric part of the profile. There is very strong correlation between radial velocities of emission and absorption components. At mean the radial velocity of emission is somewhat higher than the radial velocity of absorption (see Figure 2c).

The measured $V / R$ relations of the $\mathrm{H}_{\alpha}$ and $\mathrm{H}_{\beta}$ lines are plotted in the right part of Figure 2. We can see that in the most cases the $V / R<1$ (filled circles) for $H_{\alpha}$ and $V / R>1$ (open circles) for $\mathrm{H}_{\beta}$. The violet $(\mathrm{V})$ peak of the $\mathrm{H}_{\alpha}$ line was first time higher than the red (R) peak $(\mathrm{V} / \mathrm{R}>1)$ in the middle of January 2007. For both lines the deep minimum took place in 2005, directly before the starting of shell phase formation. During the years of 1999-2002 there has been a remarkable trend that V/R was increased. At the same time the fluctuation was quite large. Our observations show that there is a good correlation between the variations of $\mathrm{V} / \mathrm{R}$ relations of the $\mathrm{H}_{\alpha}$ and $\mathrm{H}_{\beta}$ lines.

\section{Acknowledgements}

This work was supported by the target-financed project SF0060030s08 financed by the Ministry of Education and Research of Estonia. 